\title{
¿Una orientación marcada por el género? El caso de la Formación Profesional Básica valenciana
} An Orientation Marked by Gender? The Case of the Valencian Basic Vocational Education and Training

\author{
Sandra Obiol-Francés, Elisabet Almeda Samaranch, Dino Di Nella, Nuria Pumar Beltrán, \\ Aida Ruiz Franco, Núria Vergés-Bosch y Alícia Villar-Aguilés ${ }^{1}$
}

\begin{abstract}
Resumen
En este artículo presentamos parte de los resultados obtenidos en el análisis realizado a la Formación Profesional Básica desde una perspectiva de género, un nuevo ciclo formativo incluido en la etapa obligatoria del sistema educativo español con el fin de evitar el abandono educativo precoz. Con este objetivo se conjuga adaptación y diversificación curricular puesto que el curriculum académico se estrecha en pro de un mayor contenido de carácter profesionalizador según ramas ofertadas. En este caso el género emerge como variable fundamental para entender el escaso número de mujeres matriculadas, así como su concentración en ramas claramente connotadas en términos de género. Las entrevistas realizadas a los equipos de coordinación y orientación, fundamentales en el acceso a una FPB, nos permiten constatar la pervivencia de una segregación por género de los diferentes itinerarios formativos justificada sobre las preferencias individuales. Esta justificación conlleva tomar medidas desde los centros en términos únicamente individuales y siempre mediatizadas por los escasos recursos (personales y formativos) disponibles, pero sobre todo por la que es su principal prioridad: evitar el abandono precoz de los estudios. El resultado es un ciclo formativo en el que las mujeres están, o bien ausentes, o bien concentradas en ciclos fuertemente feminizados, disminuyendo sus oportunidades formativas y laborales.
\end{abstract}

\section{Palabras clave}

Género, abandono educativo temprano, formación profesional, segregación formativa/ocupacional por género, elecciones educativas.

\section{Abstract}

In this article we present part of the results in the analysis of the Basic Vocational Education and Training from a gender perspective. A new training cycle included in the compulsory stage of the Spanish educational system in order to avoid early educational abandonment. To this end, adaptation and curricular diversification are combined since the academic curriculum is narrowed in favor of a greater professional content according to the branches offered. Gender clearly emerges as a fundamental variable to understand the small number of women enrolled, as well as their concentration in branches specially connoted in terms of gender. The interviews carried out with the coordination and orientation teams, which are essential for access to a FPB, allow us to verify the persistence of a gender segregation of the different formative itineraries justified on individual preferences. This justification implies taking measures from the centres in only individual terms and always mediated by the scarce available resources (personal and training). But, above all for what is their main priority, that is to avoid the early abandonment of studies. The result is a training cycle in which women are either absent or concentrated in strongly feminized training cycles, reducing thus their training and employment opportunities.

\section{Keywords}

Gender, early school leaving, Vocational Education and Training, gender educational/occupational segregation, educational choices.

\section{Cómo citar/Citation}

Obiol-Francés, Sandra; Almeda Samaranch, Elisabet; Di Nella, Dino; Pumar Beltrán, Nuria; Ruiz Franco, Aida; Vergés-Bosch, Núria y Villar-Aguilés, Alícia (2020). ¿Una orientación marcada por el género? El caso de la Formación Profesional Básica valenciana. Revista de Sociología de la Educación-RASE, 13 (3), 371-391. http://dx.doi.org/10.7203/RASE.13.3.16666.

\footnotetext{
1 Sandra Obiol-Francés, Universitat de València, sandra.obiol@uv.es; Elisabet Almeda Samaranch, Universitat de Barcelona, elisabet.almeda@ub.edu; Dino Di Nella, Universidad Nacional de Río Negro, dino.dinella@unrn.edu.ar.; Nuria Pumar Beltrán, Universitat de Barcelona, npumar@ub.edu; Aida Ruiz Franco, Universitat Pompeu Fabra, aida.ruiz@upf.edu; Núria Vergés-Bosch, Universitat de Barcelona, nu.verges@gmail.com; Alícia Villar-Aguilés, Universitat de València, alicia.villar@uv.es.
} 


\section{Introducción}

En el Estado español, la tasa de abandono escolar temprano, es decir, la proporción de jóvenes entre 18 y 24 años que no han completado la educación secundaria superior ni tampoco estudian ni reciben formación alguna, alcanza el 17,2\% en 2019 una cifra que continúa siendo inusualmente elevada pese a que ha descendido en los últimos años coincidiendo con la crisis económica. De hecho, España es el primer estado de la Unión Europea con la tasa más alta de abandono escolar, seguido de Malta con un 16,7\% (Eurostat, 2020) y el número de jóvenes que no están ni empleados ni en educación o formación supera ampliamente el promedio de la OCDE (OECD, 2018: 4), siendo de los más elevados de la Unión Europea con un 12,4\% (Eurostat, 2020).

En estas cifras, las diferencias entre hombres y mujeres son significativas pues un 21,2\% de los hombres jóvenes abandonan los estudios frente al 13\% de las mujeres (Eurostat, 2020), una diferencia de más de 8 puntos porcentuales, la mayor de toda la Unión Europea. Aunque ambas cifras, tanto la referida a las mujeres como a la de los hombres, superan el objetivo que se había propuesto para 2020 en el Marco estratégico para la cooperación europea en el ámbito de la educación y la formación (ET 2020)를 en el centro del discurso político y mediático acerca del desenganche, y finalmente, abandono educativo, se ubica mayormente a los chicos, no a las chicas. No en vano, la repetida idea del éxito educativo de las mujeres, avalado por el gran incremento de la presencia de las mujeres en todos los ciclos educativos, puede enmascarar una realidad igualmente constatada por las cifras: la existencia de un volumen significativo de chicas que se desenganchan del sistema educativo. No centrar nuestra mirada en estas mujeres conduce a no entender a las mujeres como un colectivo de atención prioritaria en las agendas políticas respecto el abandono escolar precoz y en consecuencia perjudica gravemente sus oportunidades futuras en el mercado de trabajo. Precisamente nuestra propuesta es centrar la mirada en el género como elemento explicativo de la relación que los y las jóvenes mantienen con el sistema educativo en nuestro país.

En la investigación que da origen a este artículo $^{3}$, nuestro principal objetivo ha sido conocer la incidencia del género en la construcción de trayectorias educativas, centrándonos en aquellos itinerarios próximos al abandono de la educación reglada. Más en concreto nos interesa conocer en qué medida el género contribuye a optar por itinerarios creados expresamente como medida de contención al abandono, así como si existe una asimetría de género en su interior. Para ello nos hemos centrado en el estudiantado que se encuentra cursando Formación Profesional Básica (de ahora en adelante: FPB), una reciente y controvertida medida de política educativa establecida en el Estado español para luchar contra el riesgo de abandono precoz de los estudios desde la implantación de la Ley Orgánica 8/2013 para la Mejora de la Calidad Educativa (LOMCE) que se introduce a partir del curso 2014-2015 con el fin de reducir el abandono educativo, en un contexto y un momento de altos niveles de este fenómeno socioeducativo. Vino a sustituir a los antiguos Programas de Cualificación Profesional Inicial (PCPI) manteniendo su principal objetivo: identificar al colectivo de estudiantes en riesgo de desenganche del sistema educativo y ofrecerles una alternativa curricular para evitar su abandono (Roca, 2010: 59). Para ello, la FPB incluye, todavía en la etapa de educación obligatoria, diferentes familias profesionales a través de las cuales el estudiantado puede acceder, al finalizar el ciclo, a una certificación con validez académica y profesional ${ }^{4}$.

2 Esta agenda marcó como objetivo para el abandono educativo prematuro no superar el $10 \%$ entre los y las jóvenes de 18 y 24 años.

3 «Género y decisiones educativas. Construcción de itinerarios formativos en la Formación Profesional Básica» financiada por el Centro Reina Sofía sobre Adolescencia y Juventud (2018-2019).

4 Cualificación de nivel 1 del Catálogo Nacional de las Cualificaciones Profesionales (Ley Orgánica 5/2002, de 19 de junio, de las Cualificaciones y de la Formación Profesional, artículo 7). 
Sin embargo, siguiendo a Bonal, Tarabini y Verger (2015), supone una vuelta atrás en los objetivos inclusivos establecidos por la LOGSE al dirigir a estudiantes más jóvenes hacia un itinerario secundario al tiempo que restringe futuras trayectorias profesionales. Al tiempo que supone relacionar la formación profesional de nuevo con el riesgo de abandono educativo, anulando los esfuerzos realizados para evitar esta vinculación (Merino, 2012).

Nuestro propósito en la investigación realizada ha sido analizar la FPB desde una perspectiva de género puesto que contiene, a nuestro entender, dos elementos significativos que reclaman esta mirada. En primer lugar, el acceso a este itinerario formativo se basa en unos requisitos entre los que está haber sido propuesto a progenitores o tutores legales por el equipo docente (Real Decreto 127/2014, de 28 de febrero, BOE, núm. 55 de 5 de marzo de 2014). Es decir, que se trata de un acceso vehiculado de manera directa por los mismos centros educativos y eso lo hace sumamente interesante para conocer el peso que tiene el género, y también otras variables estructurales, en la propuesta que se realiza al estudiantado de acceder a un itinerario de FPB. En segundo lugar, en su afán por aportar un carácter práctico y profesionalizador, esta medida se convierte en un nuevo ciclo de Formación Profesional y por tanto requiere de la elección de una determinada familia profesional: queríamos conocer cómo influye el género en esta elección, que puede venir a significar optar por un sector laboral.

En definitiva, nuestro interés en este artículo es acercarnos a este acceso a la FPB a través de entrevistas realizadas a docentes. De entrada, los datos nos muestran que las chicas son clara minoría (un 28,9\% de la matrícula en el curso 2016-17). Y, sobre todo, nos interesa conocer la actitud de los docentes ante la segregación por género que se hace evidente en los datos de matriculación en las diferentes opciones formativas que pueden cursarse en FPB y las posibles medidas que se toman desde los centros para evitar esta segregación. Y lo hacemos en un territorio muy concreto: el País Valenciano, con un porcentaje de abandono educativo temprano por encima de la media estatal (un 20,3\% en 2017 frente a un 18,3\%). No es extraño que sea uno de los territorios españoles con mayor número de alumnado matriculado en FPB así como de centros que la imparten, si exceptuamos Andalucía. Los datos nos muestran que en el curso 2016-17 del alumnado matriculado en FPB en España, un 15,5\% se localizaba en el País Valenciano, así como un $16,2 \%$ del total de centros que impartían estas enseñanzas.

El documento se divide en cuatro partes. En primer lugar, enmarcamos teóricamente nuestro propósito investigador: trataremos de explicar la segregación de género existente en el mercado de trabajo entre diferentes perfiles profesionales y sectores entendidos de manera generalizada como «propios» de mujeres y hombres, y la relación que esta segregación mantiene con unas trayectorias formativas igualmente encuadras por expectativas de género. En segundo lugar, exponemos la metodología seguida para llevar a cabo nuestro análisis y continuamos con el análisis de los resultados en los que hemos diferenciado el acceso del estudiantado a la FPB y la elección de familia profesional. Concluimos el texto con la constatación de la existencia de una asimetría de género de los itinerarios formativos pero su reversión se subordina en los centros al objetivo de evitar una situación de abandono precoz de los estudios.

\section{Segregación de género en la ocupación y la formación}

Desde hace décadas las investigadoras feministas, así como los datos oficiales respecto a la ocupación, nos alertan de las profundas desigualdades de género que se mantienen en el mercado de trabajo; se observa aún una profunda división sexual del trabajo, tanto vertical como horizontal (Torns y Recio, 
2012; Burchell et al., 2015; González, Vergés y Martínez, 2017; Cebrián y Moreno, 2018; OIT, 2018). El estado actual de la segregación laboral en Europa muestra que las mujeres están más presentes en ocupaciones dominadas por mujeres (un 69\% en ocupaciones donde se supera el 60\% de mujeres) y apenas se encuentran en las ocupaciones dominadas por hombres (un 13\%) (Burchell et al, 2015). Persiste una segregación ocupacional que implica que los hombres siguen siendo mayoría en los trabajos y sectores más formalizados, mejor remunerados, con mayor reconocimiento social, con poder y con potencial de futuro, como por ejemplo los sectores relacionados con las tecnologías (Castaño, 2005; Barberà, Candela y Ramos, 2008; Vergés, 2012; Castaño y Webster, 2014; González, Vergés y Martínez, 2017). En cambio, las mujeres se encuentran sobrerrepresentadas en los trabajos relacionados con los cuidados. Este tipo de trabajos gozan de peores condiciones laborales, derechos, remuneración, prestigio y posibilidades de movilidad social y progreso futuro (Ibáñez, 2008; Torns y Recio, 2012; Durán, 2018). Y, además, la participación laboral de las mujeres es menos diversa que la de los hombres. En el caso español, la mitad del empleo femenino se concentre en diez actividades profesionales frente a las dieciséis de los hombres (CES, 2016).

Estrechamente relacionada con esta división sexual del trabajo nos encontramos con una clara segregación que también se da en la esfera formativa. Aunque en los últimos años la presencia de las mujeres en todos los niveles académicos ha aumentado de manera notable hasta devenir la mayoría de personas egresadas universitarias, aún resultan en una alarmante minoría en lo relacionado con las ingenierías y la tecnología tanto en estudios de grado como de postgrado (Obiol y Villar, 2016; MECD, 2018; INE, 2018). En el proceso de elección de estudios universitarios las diferencias entre hombres y mujeres se identifican en las razones de elección del grado y también en la utilización de recursos para obtener más información sobre la oferta de titulaciones (Villar y Hernàndez, 2014). Si atendemos a la formación profesional, las mujeres siguen siendo una minoría en el estudiantado y esta segregación no solo se repite, sino que se agrava. Aunque todavía es considerado un itinerario formativo menor (Merino y Martínez, 2012; Merino, 2012; Villar, 2017), la formación profesional podría acabar generando mejores opciones laborales para los hombres que para las mujeres y con ello, agudizando las desigualdades de género existentes (Millenaar, 2014; Niemeyer \& Colley, 2015; Høst, Seland, Skålholt, 2015).

La investigación específica para el caso de la elección de formación profesional en relación al género sigue siendo escasa, los resultados apuntan también a este tipo de factores estructurales y de estereotipos. Los estereotipos de género forman parte de nuestra socialización y se reproducen entre estudiantes, profesorado, familias, medios de comunicación e instituciones públicas de forma que dan continuidad y se retroalimentan con la segregación formativa y ocupacional existente (Subirats y Tomé, 2007; Frutos, 2010; Sáinz, 2011; Sáinz, Palmen y García-Cuesta, 2012; Vázquez-Cupeiro, 2015; Casado, Meneses y Sancho, 2016, Subirats, 2016). En este sentido, tanto la selección como la oferta de itinerarios formativos siguen estando fuertemente estereotipados por género y pueden generar unas peores opciones de futuro para estas chicas y para chicos no normativos en el género masculino. Los estereotipos de género atribuyen características, comportamientos y roles a mujeres y hombres de forma binaria y diferenciada. En base a ello, las personas otorgan valor a las actividades y comportamientos humanos e influencian las expectativas educativas y laborales de chicas y chicos (Vázquez-Cupeiro, 2015). De este modo, tanto las preferencias, como incluso buena parte de la oferta y orientación de formación ocupacional para las chicas siguen basándose en la creencia que las mujeres son afectivas, empáticas, intuitivas, dependientes y pasivas y apuestan por itinerarios profesionales feminizados, por ejemplo, los relacionados con los cuida- 
dos personales, de los niños y/o del hogar y los enfermos, o de tareas administrativas. Si bien no se observan discriminaciones de género explícitas e institucionalizadas como antaño, el sistema de formación profesional actual parece aún adolecer de una ceguera de género en su aplicación que dificulta realizar transformaciones importantes (Fernández e Ibáñez, 2018).

Los estereotipos de género no solo afectan a las decisiones formativas y laborales que toman las mujeres y hombres, así como las orientaciones que reciben por parte de sus familias, profesorado, medios de comunicación y entornos de amistades, sino que se encuentran en las raíces del sistema, también educativo y laboral, que tiende a actuar con ceguera de género y reproducir, así, estructuras heteropatriarcales. De hecho, podemos considerar a las instituciones educativas como entornos de género donde se reproducen las jerarquías de poder. En este sentido la institución educativa puede ser entendida como un agente institucional promotor de estructuras y de regímenes de género (Connell, 2001).

Por otra parte, consideramos necesario en esta investigación partir de la perspectiva que contempla a las instituciones educativas y los espacios laborales como organizaciones con marca de género o generizadas (gendered organizations, Acker, 1992), en este sentido se llevan a cabo prácticas de género en entornos educativos y también laborales que refuerzan las expectativas hegemónicas de género en un sentido que conducen a las mujeres a asumir mayormente las responsabilidades de crianza y cuidados (Trotter, 2017). Estas prácticas mantienen el discurso y la representación social de «trayectorias formativas y profesionales de chicas» $\mathrm{y}$ «trayectorias formativas y profesionales de chicos». Lo que nos preocupa al respecto es que todo ello está profundamente relacionado con la elección de estudios que llevan a cabo chicas y chicos, y específicamente en la formación profesional.

Ante la implementación de una nueva formación básica para mejorar las posibilidades laborales y vitales de personas que parecían estar destinadas a abandonar la formación obligatoria es crucial una evaluación de su implementación y la generación de investigaciones que complementen la escasa información al respecto, sobre todo, si tenemos en cuenta el género. Así, es necesario que nos preguntemos qué factores operan en las elecciones de trayectorias formativas y profesionales de las mujeres y cuáles son la oportunidades presentes y futuras que les ofrecemos en nuestra sociedad. Urge pues, seguir avanzando en el conocimiento la incidencia del género y la formación profesional, así como sus contenidos, funcionamiento y las decisiones educativas y profesionales que hay detrás.

\section{Metodología}

La investigación en la que se basa este artículo ha tenido un marcado carácter cualitativo puesto que nuestro propósito era poder comprender mejor las trayectorias del estudiantado hacia la FPB, tanto como medida de retención en el sistema educativo en casos de riesgo por abandono, y hacerlo desde una perspectiva de género. Y al tiempo buscábamos que los diferentes agentes sociales implicados (docentes, jóvenes y familias) nos pudieran narrar sus percepciones acerca de este trayecto, así como los motivos de su elección por una especialidad concreta. Para ello contactamos con un total de siete centros educativos en la Comunidad Valenciana que impartían FPB (consultar tabla I, anexo), elegidos según los siguientes criterios:

a) tamaño del hábitat: procuramos evitar la ciudad de València (que concentra el 11,42\% de los centros que imparten FPB en el curso 2019-20) ya que queríamos explorar la incidencia de contar con, o no, de opciones reales de elección para el alumnado. Al tiempo nos interesaba conocer, aunque 
no lo hemos tratado en este artículo, el peso del control social en las decisiones basadas en estereotipos de género, probablemente más evidente en poblaciones de tamaño reducido.

b) existencia de itinerarios fuertemente masculinizados/feminizados en el mismo centro.

c) y, por último, la titularidad del centro, una variable fundamental que nos remite a la capacidad del centro de contar con determinados recursos a la vez que a la defensa de determinados valores sociales. Aunque hemos de tener en cuenta que su incidencia en la FPB es escasa: únicamente un 8,71 \% de los centros valencianos que imparten este ciclo son de titularidad privada (curso 2016-17, Ministerio de Educación y Formación Profesional).

Para acceder a los centros nos hemos apoyado en contactos sindicales y personales, además, hemos contado con el apoyo de la Dirección General de Formación Profesional y Enseñanzas de Régimen Especial de la Conselleria d'Educació, Investigació, Cultura i Esport de la Generalitat Valenciana. Cabe señalar que, como ocurre habitualmente en investigación social, realizamos trabajo de campo en aquellos centros que aceptaron participar. Y que, a pesar ningún centro de los que contactamos declinó participar, la misma toma de contacto es un sesgo a tener en cuenta. Es decir, que incluimos a centros con una mínima sensibilidad por el tema o al menos por ofrecer información a investigadores académicos.

En conjunto el trabajo de campo en los centros educativos se ha configurado con información cuantitativa recopilada sobre los programas formativos implementados junto a la realización de entrevistas grupales al equipo directivo, tutores/as, orientadores/as y coordinadores/as de igualdad, además de entrevistas en profundidad a madres y padres del estudiantado de FPB, entrevistas grupales a chicos y chicas cursando $2^{\circ}$ de ESO y, como elemento central, 35 entrevistas en profundidad a chicas y chicos que estaban cursando FPB. Además, una vez finalizada la investigación, llevamos a cabo diversos talleres coeducativos en la mayor parte de los centros analizados con el fin doble de promover buenas prácticas en términos de igualdad de género ${ }^{5}$ y poder obtener mayor información sobre los procesos de decisión sobre sus itinerarios formativos y sobre el peso del género en sus actitudes y comportamientos.

En este artículo nos centramos en el análisis de las entrevistas realizadas a los equipos de dirección y orientación. Estas entrevistas fueron la primera toma de contacto con los centros y las llevamos a cabo en cinco de los centros a los que accedimos. Fueron un total de 5 entrevistas grupales en las que estaban presentes miembros del equipo de dirección, los orientadores/as y las coordinadoras de igualdad y convivencia. Las entrevistas se realizaron en el mismo centro educativo, generalmente en el despacho de coordinación/dirección, durante horario lectivo, siendo todas entrevistas largas, de más de 2 horas. El guion constaba de cuatro apartados: valoración del funcionamiento del programa de FPB, descripción del proceso por el cual se decide en el centro que chicas y chicos accedan a cursar una FPB, descripción del perfil del estudiantado y de las razones por las que llegan a una FPB, y, por último, acciones en pro de una mayor igualdad de género que se lleven a cabo a nivel de centro y a nivel del ciclo de FPB. Las entrevistas se realizaron a lo largo de los meses de octubre a diciembre de 2018 y fueron grabadas, transcritas, codificadas utilizando el software MAXQDA y, finalmente, analizadas. A continuación, pasamos a presentar algunos de los resultados obtenidos en esta investigación.

5 Vergés-Bosch, Núria; Freire, León; Obiol-Francés, Sandra (2019) «Claves para la atracción y permanencia de las mujeres en la formación profesional TIC». 


\section{Formación Profesional Básica: itinerarios con marca de género}

\subsection{La segregación de las elecciones formativas de Formación Profesional Básica en datos}

Los datos disponibles acerca de las diferentes elecciones formativas que toman chicas o chicos nos muestran una realidad claramente asimétrica, especialmente si ponemos el foco en el contenido de los estudios que se realizan. Es en el inicio de la educación postobligatoria cuando la separación empieza a ser clara, siendo las chicas las que en mayor medida optan por cursar Bachillerato (presencial): un $54,2 \%$ del total de matrículas realizadas para el caso valenciano en el curso 2016-17 (un 52,7\% en el caso estatal) mientras que este peso porcentual se invierte en pasar a referirnos a la Formación Profesional en su conjunto ${ }^{6}$. Esta mayor tendencia de las chicas que los chicos hacia itinerarios de carácter más académico se ha mantenido en el tiempo (Martínez y Merino, 2011).

En el curso 2016-17, los ciclos formativos de grado medio del sistema valenciano las mujeres vienen a representar un 43,3\% del total de estudiantes matriculados (42,3\% en el conjunto español); y para los ciclos superiores presenciales ellas ascienden a un 47,1\%, aunque en la opción de formación a distancia las mujeres alcancen una cifra muy elevada (un 60,2\%, un 59,5\% la media española). Es precisamente en la FPB donde se produce una mayor asimetría entre hombres (69,5\%) y mujeres (30,5\%), la cual es menor en el caso valenciano que en el español (71,1\% y 28,9\%). Es importante señalar la circunstancia que al tratarse la FPB de una etapa de la educación obligatoria estaríamos viendo cómo se traslada la asimetría de género a la etapa central de nuestro sistema educativo, como también la capacidad de la FPB para plantear nuevas oportunidades para la reorientación vital y laboral (Cacheiro, García y Moreno, 2016).

Como decíamos, la diferencia de género entre itinerario académico y profesional no es nueva. Observemos la información que nos proporcionan los datos valencianos respecto a esta diferencia. En primer lugar, en el curso 2016-17, la tasa bruta de finalización de la ESO era de un 73,2\% entre las chicas y de un $60,6 \%$ entre los chicos, casi 13 puntos porcentuales de diferencia. Encontramos una diferencia ligeramente mayor en la tasa bruta de finalización de Bachillerato en el mismo curso, que es de un $43 \%$ en el caso de los chicos y un 57,4\% en las chicas. En sentido contrario la diferencia se torna a favor de los chicos al fijarnos en el mismo indicador en el caso de la FPB: un $8 \%$ de los chicos frente a un $4,1 \%$ en el caso de las chicas en los centros valencianos durante el curso 2016-17. Es decir, que los chicos transitan un camino menos académico podríamos decir que no las chicas, y que el discurso del éxito académico de las chicas funciona a través de estas cifras. Por otro lado, la tasa de idoneidad entre edad y curso a los 15 años es más elevada en el caso de las chicas $(71,9 \%)$ que no en el de los chicos $(62,2 \%)$. Y se aprecia también que en la promoción curso a curso en la ESO son los chicos quienes van abandonando en mayor medida que las chicas: en el curso 2016-17, si comparamos el total de chicos matriculados en 1 er curso con los de $4^{\circ}$ curso se observa una disminución de un 32,05\% mientras que entre las chicas es del 20,22\%, demasiada diferencia para atribuirla únicamente a razones demográficas. Ante la falta de datos concretos por género sobre alumnado con bajo rendimiento académico, que sería el colectivo que accedería en mayor medida a la FPB, los indicadores relacionados nos pueden ayudar a comprender que la presencia minoritaria de las mujeres en este ciclo puede provenir de una mayor propensión de las chicas a optar por un itinerario de carácter más académico.

6 Datos basados en las estadísticas del Ministerio de Educación y Formación Profesional. 
Esta asimetría que hemos encontrado es muy visible en la opción que los y las jóvenes muestran por una u otra familia profesional. La oferta de familias profesionales que se incluyen en la FPB en el Estado español son las siguientes: Actividades Físicas y Deportivas, Administración y Gestión, Agraria, Artes Gráficas, Comercio y Marketing, Edificación y Obra Civil, Electricidad y Electrónica, Fabricación Mecánica, Hostelería y Turismo, Imagen Personal, Industrias Alimentarias, Informática y Comunicaciones, Instalación y Mantenimiento, Madera, Mueble y Corcho, Marítimo-Pesquera, Servicios Socioculturales y a la Comunidad, Textil, Confección y Piel, Transporte y Mantenimiento de Vehículos y Vidrio y Cerámica. Estas familias están implantadas casi en su totalidad en el sistema valenciano, solamente dos de ellas (Actividades Físicas y Deportivas y Servicios Socioculturales y a la Comunidad) no se ofertan por el momento.

Si consideramos que el intervalo para considerar la feminización/masculinización de las familias profesionales se encuentra entre el $40 \%$ y el $60 \%$, tal y como hace la Unión Europea en el caso de las profesiones, podríamos decir a la luz de los datos disponibles, que únicamente Imagen Personal con un $83,4 \%$ (curso 2016-17) ${ }^{7}$ es una especialidad en FPB que podríamos considerar feminizada. Y que solo cinco itinerarios de los 17 existentes en el País Valenciano podrían considerarse mixtos: Textil (58,2\%), Comercio y Marketing (54\%), Administración y Gestión (53\%), Artes Gráficas (45,3\%) e Industrias Alimentarias (41,8\%). El resto son itinerarios masculinizados, algunos de manera muy acentuada como es el caso de Transporte y Mantenimiento de Vehículos con tan solo 1,9\% de estudiantes matriculadas, y Fabricación Mecánicay Electricidad y Electrónica en las que un 4,3\% de las matrículas pertenecen a mujeres.

En definitiva, no solo estamos ante un ciclo formativo que está muy masculinizado, sino que las escasas chicas que se matriculan lo hacen en un número muy reducido de ramas profesionales, entre los cuales destaca precisamente aquel que remite a preocupaciones tradicionalmente adscritas a las mujeres, en este caso el cuidado y la belleza física.

\subsection{Un acceso asimétrico a la Formación Profesional Básica}

Las chicas matriculadas en FPB, como hemos señalado, apenas superan un tercio del total de matrículas realizadas en los centros valencianos donde imparten este ciclo formativo. Lejos de considerarla una diferencia de capacidades entre chicas y chicos, los equipos de dirección y orientación de los centros entrevistados se refieren constantemente a la actitud ante los estudios y las normas de funcionamiento del centro educativo, ahora sí, diferente entre chicas y chicos, verbalizando la «marca de género» de la misma institución (Acker, 1992). También surge la idea de que son las expectativas de los docentes y de las familias las que obstaculizan el acceso de las chicas a la FP en general, y en especial a la FPB, consideradas todavía como itinerarios alternativos de menor rango (Villar, 2017) y sobre todo se evita que accedan a ramas profesionales muy masculinizadas:

«Potencialmente son exactamente iguales. Es por la perversión de la orientación desde primaria a secundaria. Es decir, a la mujer se le da una protección que está fuera de lugar, y se le cortan las posibilidades de orientar directamente a FP, a ramas a disciplinas de FP consideradas de hombres». C2. ${ }^{8}$

\footnotetext{
Elaboración propia a partir de datos del Ministerio de Educación y Formación Profesional.

8 A pesar de que muchas de las entrevistas incluidas en la investigación se realizaron en catalán, hemos decidido traducir directamente los extractos incorporados en el texto para facilitar su lectura.
} 
Se trata de estudiantes, en la práctica totalidad de las ocasiones, con trayectorias académicas convulsas, podríamos decir. Estudiantes que han repetido curso en más de una ocasión, que han sido expedientados por mal comportamiento con frecuencia, que, en definitiva, docentes (y familias) conciben la relación de estos y estas jóvenes con el sistema educativo como problemática y como parte de la denominada cultura anti-escuela. Por eso se les ofrece la opción de cursar una FPB, incluso podríamos decir que se les dirige hacia la misma. Y en este caso, según las entrevistas realizadas, son pocas las chicas que no se adaptan al funcionamiento cotidiano establecido en el centro y eso conlleva que, independientemente de los resultados académicos, los chicos tengan una mayor tendencia a acceder a opciones alternativas al núcleo central de la formación reglada. Para las personas entrevistadas las chicas son más aplicadas en sus estudios y muestran un mejor comportamiento. Cumplen, en definitiva, con el rol atribuido históricamente a las mujeres por el que son más calladas, más obedientes, más disciplinadas. Al contrario que en el caso de los chicos donde es mucho más frecuente que protagonicen conflictos en el centro, que muestren agresividad o reten al profesorado, según nos describen en las entrevistas realizadas. Los estereotipos de género que marcan las trayectorias formativas, y después laborales, como hemos indicado en el apartado teórico, son evidentes en el acceso a la FPB.

Se identifican dos caminos de acceso a la FPB: malos resultados académicos pero una conducta no disruptiva en el aula (que en absoluto significa que no se trate de una actitud apática y que el estudiantado no muestre un desenganche total con los estudios); y, en segundo lugar, malos resultados académicos combinado con importantes problemas convivenciales en el centro. Las chicas son minoritarias en ambos, especialmente en esta última opción, según se nos narra en las entrevistas realizadas. Sin embargo, las hay. Son las chicas consideradas como «problemáticas» o «gamberras», las ladettes que conceptualizan desde la Sociología británica (Jackson, 2006). Y cuando nos encontramos con ellas la percepción de los docentes y orientadores entrevistados es diferente si se trata de una chica o de un chico. Se les atribuye una actitud más retorcida, más compleja además de mediatizada por la figura de los chicos. No es extraño: la ruptura con las expectativas de género es mayor y, por tanto, genera un mayor rechazo. Son desviaciones a las normas, que en el caso de las mujeres son más duramente castigadas, tal y como hemos comprobado en investigaciones anteriores (Cantalapiedra, Marginet y Almeda, 2019).
«La problemática de las chicas es diferente. [...] El nivel de madurez de las chicas es diferente al nivel de madurez del chico. El chico está pensando, su testosterona es un armario ropero, pero cuan- do dices: Eh, quieto. Quieto y responde. La chica es más complicada, tiene otro tipo de problemas, yo alucino en la época en la que estamos muchas de ellas dependen del novio». C2.

Por supuesto, las variables de clase social y etnia aparecen en las entrevistas para explicarnos por qué chicas y chicos se encaminan a una situación de desenganche educativo. A pesar de que cada vez más, nos dicen, acceden a la FPB chicas y chicos de entornos que no presentan, al menos por sus narraciones, dificultades económicas y relacionales, sino que están incómodos con la dinámica habitual en la formación reglada, la gran mayoría provienen de un contexto familiar y económico en el que identifican numerosos obstáculos para poder seguir el ritmo académico marcado por el centro. 


\subsection{Elección diferenciada de itinerarios profesionales}

\section{(a) Un sistema con opciones muy limitadas}

Las cifras expuestas con anterioridad se reflejan con claridad en la cotidianidad de los centros. Especialmente en los centros a los que hemos accedido esta diferencia es muy visible pues ofertan ciclos claramente asimétricos por género dado que era una de nuestras premisas en la construcción de la muestra. La postura general del profesorado entrevistado es asumir resignadamente la existencia de esta asimetría entendiendo que no es más que el reflejo de nuestra sociedad y en ésta, un centro educativo puede hacer poco. La idea de que tienen poca capacidad para transformar la realidad es general, pero en el caso del centro C3 surge de manera más evidente y combativa por parte de uno de sus orientadores.

«es que yo no tengo por qué sentirme culpable de cómo funciona la sociedad. ¿Está claro? [...] este es el eterno dilema de que aqui tenemos que enseñarles absolutamente todo. Lo que no se les enseña en la sociedad o la sociedad enseña de manera equivocada y torticera tenemos que compensarlo los centros educativos». C3.

En su discurso se evidencia con rotundidad un agotamiento ante las frecuentes propuestas de acción por el cambio social focalizadas en la educación, que viene a significar en la escuela y en sus docentes. Nos hemos encontrado con equipos directivos exhaustos, sobre todo al tratar con centros que han liderado cambios significativos en sus proyectos metodológicos y que además tienen unas dimensiones considerables con multitud de ciclos formativos ofertados y por tanto con multitud de problemáticas diferentes que afrontar.

$\mathrm{Al}$ apelar a las personas entrevistadas sobre los factores que intervienen en la elección por parte del estudiantado de la especialidad de FPB, surge con claridad la capacidad de influencia que tienen sobre los jóvenes sus iguales, incluso llegan a señalar cómo todo el trabajo que se pueda hacer desde el centro para romper con estereotipos de género, pero también para evitar posibles abandonos educativos, chocan con el muro de la aceptación de sus compañeros y compañeras: «Porque el otro día yo hablaba con uno de carpintería y me decía: Es que yo quería haber elegido peluquería, pero claro, todo el mundo decía: ¿Tú peluquería?» C4. Aparecen otros factores a tener en cuenta como es el peso de los medios de comunicación para que se conozcan más o menos algunos perfiles profesionales, o el peso de los estereotipos de género que las familias imponen con mayor o menor fuerza, pero que siempre están presentes a pesar de que el estudiantado reclama su absoluta libertad en la elección como ya hemos identificado en otras etapas formativas (Obiol, 2017).

«es que las propias familias, ¿cómo te vas a meter en automoción? O sea, si no puedes ni coger la bolsa de la compra, ¿cómo vas a levantar un motor o levantar un coche?». C3.

Además de los factores externos que para las personas entrevistadas son los más relevantes hemos de tener en cuenta el perfil del estudiantado al que nos estamos refiriendo en conjunción con la organización de los estudios en los que centramos nuestro interés. Pero al mismo tiempo estamos hablando de estudiantes con un perfil muy concreto. Son muy 
jóvenes ${ }^{9}$ y generan una escasísima confianza en que sean capaces de responsabilizarse de su vida cotidiana, de la asistencia regular al centro, incluso. Este perfil, descrito por los docentes entrevistados pero que también hemos visto reflejado en las entrevistas realizadas a los mismos jóvenes y a las familias (Villar y Obiol, 2020), coincide con una escasa planificación territorial de las familias profesionales que se implantan en los diferentes centros. En muchas ocasiones la decisión de implantar una especialidad de FPB viene más determinada por el perfil del equipo docente con el que cuentan o por la dificultad de dotar a los centros para impartir determinados ciclos que requieren contar con maquinaria y espacio muy costosos de conseguir, que no con las necesidades y demandas del territorio en el que se ubican. Y eso unido a las dificultades que genera un territorio como el valenciano, deficitariamente conectado con transporte público. En definitiva, la mayoría de las veces la decisión por matricularse en un ciclo o en otro no viene por las preferencias sino por qué posibilidades tienen para elegir, que casi siempre son muy escasas: acaban eligiendo lo que tienen más a mano, lo que supone un riesgo menor de perder el control del estudiantado.

«Y luego viene aquello de ajustarse a: No tengo otra alternativa, ¿por qué? Pues porque si soy consciente de que me cuesta y tal, pues no tengo más remedio que adaptarme a lo que me han dado, a lo que me ban puesto, a lo que tengo. Y una cuestión fundamental es la lejanía, la oferta es fundamental [...] Y claro dices: ¿Qué hago con la criatura? Entre no estudiar, pues que estudie algo, aunque sea aquì. C3.

Incluso aparece, de manera significativa, que la oferta de especialidades de FPB adolece de una importante masculinización, con apenas ciclos que podrían encuadrarse en el espacio tradicionalmente considerado femenino: «en la [Formación Profesional] Básica, ahora mismo las chicas tienen menos donde elegir si nos centramos en lo tradicionalmente elegido por ellas» C5. Las instituciones educativas, tal y cómo señalábamos citando a Connell (2001) promueven estructuras y regímenes de género.

Y esta postura nos parece significativa por su doble significado. Por una parte, porque evidencia claramente la diferenciación de formación/ocupación por género, se asume todavía que hay intereses y profesiones más de chicas y otros más de chicos. Pero también deja traslucir una escasa diversidad en la oferta educativa que pueda atraer a los jóvenes hacia nuevos perfiles formativos y laborales que puedan ser demandados en el mercado laboral, dificultando también que los jóvenes en riesgo de abandono educativo puedan huir de los espacios más precarios del mercado de trabajo. Como nos señalan Bernard y Molpeceres (2006) estos ciclos formativos alimentan la segmentación del mercado de trabajo, conformando una bolsa de mano de obra secundaria dirigida a oficios más precarios. Es decir, el riesgo inicial de exclusión de los jóvenes se consolida en el mismo programa y viene a justificar una «injusticia educativa institucionalizada» (Abiétar-López et al., 2015) limitando la posibilidad de revertir su previa vulnerabilidad educativa y social. Al introducir el género en esta aproximación a la realidad podemos ver como existe también una segmentación simultánea por la que se dirige a las mujeres a sectores muy concretos de nuestro mercado de

9 La mayoría de los y las estudiantes entrevistados en nuestra investigación tiene 16-17 años de edad. 
trabajo, especialmente aquellos servicios dedicados al cuidado especialmente precarizados (EIGE, 2017) y por tanto limitando también sus oportunidades laborales y vitales.

El discurso de las docentes y orientadoras es ligeramente más crítico con esta situación que el de los hombres, pero la profunda interiorización de las pautas de género conlleva incluso a muestras de violencia simbólica (Bourdieu, 2000; Bourdieu y Passeron, 2001) muy significativas como es el caso del centro C5 en el que se asume con naturalidad la desigualdad de mujeres y hombres en el mercado de trabajo. Sin embargo, encontramos una importante sensación de incomodidad con la fortaleza de los estereotipos sobre los que el estudiantado construye sus opciones educativas, pero también su actitud en el aula que les afecta también en su misma práctica docente cuestionando su capacidad como docentes en ciclos formativos muy masculinizados, especialmente si imparten materias técnicas.

"Yo di un año maquinaria y me decían: ¿Tú vas a explicarme cómo la motosierra? Y digo:

Sí, ¿qué pasa?'. Y dicen: ¿ Y tú lo entiendes?».C1.

\section{(b) Una lectura individual de la segregación}

Para las y los docentes entrevistados -y coincide con los datos disponibles-aquel estudiantado que rompe con las expectativas normativas de género sobre sus decisiones formativas es escaso. Esta singularidad quizás es lo que los convierte en especialmente valorados por el profesorado como estudiantes excelentes. No obstante, es importante señalar, que se incide en el hecho que a pesar de esta ruptura en las decisiones formativas no les impide cumplir con las expectativas de género en otros ámbitos de su vida cotidiana y, por ejemplo, se maquillen o se cuiden las uñas a pesar de estar cursando un itinerario tradicionalmente masculino, como se nos expone en el centro C2.

La lectura que hacen las y los docentes de esta ruptura con el mandato de género es en clave siempre individual: estudiantes valientes, con sensibilidad especial, que tienen las cosas muy claras... eso conlleva a que las acciones emprendidas por los centros para promover la igualdad de género se centren también en su dimensión individual. En primer lugar, sus principales medidas se basan en la difusión de perfiles profesionales exitosos que no han cumplido con el mandato de género: peluqueros y cocineros reconocidos, técnicas de automoción en circuitos de motociclismo, etc. Se apela constantemente a la igualdad de capacitación profesional, a la importancia de mostrar la diversidad de opciones existentes, pero siempre sin pretender intervenir en las decisiones de los jóvenes.

\footnotetext{
«Nosotros explicamos todos los ciclos, hacemos jornadas para que ellos lo vean, les ponemos vídeos de todo. No marcamos en ningún momento ninguna tendencia y si ellos en algún momento te verbalizan un ciclo determinado, tú le buscas todas las opciones y las combinaciones posibles». C4.
}

La trayectoria académica no deja de concebirse por los y las docentes entrevistados como como una decisión únicamente personal, casi como una lucha por cumplir objetivos y sueños. Por tanto, no conciben que puedan ir más allá que simplemente sugerir que hay otros 
caminos por los que poder transitar. Unas sugerencias que son mejor recibidas por parte de las chicas, puesto que los chicos son mucho más reacios a romper con lo que se espera de ellos por ser precisamente chicos. Y a veces, como señalan los/as docentes entrevistados, a los y las jóvenes les resulta liberador encontrarse en un centro nuevo, donde no los conozcan y donde se deshagan de la presión de su grupo de pares. Por tanto, aunque no sea percibido de tal manera por el profesorado entrevistado, se elige rama profesional generizada, como se elige ocupación generizada también, por normatividad social (Evans, 2006). Su consideración individual solo genera que un fortalecimiento de la segregación.

Además, los equipos de coordinación y orientación en su práctica diaria se topan con dos obstáculos fundamentales que dificulta su ejercicio docente pero también llevar a cabo posibles medidas de promoción de la igualdad de género. En primer lugar, con la dificultad en los centros públicos de conformar sus equipos docentes debido a su elevada movilidad y temporalidad. Eso les resta capacidad de incidencia y también tiempo en la formación de estos profesores para poder aplicar determinadas medidas o maneras de trabajar en las aulas. Un elemento que sufren especialmente en FPB dado que no suelen ser prioritarios para la mayoría del profesorado. En segundo lugar, con una serie de reclamaciones y de transformaciones burocráticas que les llegan desde la Administración Pública, de decisiones políticas que muchas veces ni comparten ni tienen recursos suficientes para llevar a cabo como señalan en el cambio de los programas de PCPI a FPB pero también con la reciente creación de la coordinación de igualdad y convivencia en los centros valencianos ${ }^{10}$.

Pero, en definitiva, todas las acciones enumeradas durante las entrevistas realizadas se subordinan a un elemento clave: la meta primordial de evitar el abandono educativo. El resto, incluida la igualdad de género, puede acompañar a este propósito pero no tiene la misma importancia: «Es prioritario que sean felices y que no haya un abandono escolar. Es decir, es prioritario... yo creo que nos da lo mismo que sea chico o chica o pájaro» C4. Precisamente este último extracto muestra perfectamente cómo la segregación por género de las familias profesionales de FPB les preocupa, pero no tanto como conseguir que chicas y chicos no abandonen sus estudios. Son conscientes de la existencia y pervivencia de estereotipos de género que lleva al estudiantado a elegir unas opciones formativas muy determinadas y que estas mismas elecciones conllevan que la segregación se fortalezca y se eternice. Pero esta evidencia, que les genera más o menos incomodidad, no la entienden de manera estructural por tanto la atienden únicamente de manera individual y no consiguen hacerla mermar apenas. Y, sobre todo, tienen otros problemas que por su capacidad de disrupción en el aula y en el centro educativo, pero también en la vida presente y futura de los chicos y chicas a los que están atendiendo, presentan una mayor urgencia en su solución que no una asimetría por género de los estudios que tienen totalmente normalizada.

\section{Conclusiones}

La significativa masculinización de los estudios de FPB -mayor que la de otros ciclos de formación profesional- pone en evidencia cómo de importante es analizar el acceso a este ciclo formativo desde una

10 Resolución de 1 de julio de 2016. DOCV núm. 7826 (12.07.2016). 
perspectiva de género. $\mathrm{Y}$ todavía se hace más pertinente al constatar que las escasas chicas que cursan este ciclo -sobre un tercio del total de estudiantado matriculado-, se concentran en unas pocas familias profesionales, en concreto en «Imagen Personal» en la que se supera el $80 \%$ de chicas matriculadas. No se puede obviar que el género resulta imprescindible para entender mejor los motivos de estas opciones formativas, sobre todo al estar tan relacionadas con el riesgo de abandono prematuro de los estudios. Por ese motivo, y a pesar de que clase y origen tienen un peso importante, el género ha constituido la variable básica desde la que hemos analizado los discursos del profesorado que forman los equipos directivos y de orientación, agentes fundamentales ante el acceso y la elección del estudiantado por una u otra familia profesional de la FPB.

Nuestro análisis proporciona más evidencias respecto a la ya histórica tendencia de las chicas a optar por itinerarios de carácter más académico que no profesional (Martínez y Merino, 2011; Merino y Martínez, 2012) que en este caso además significa un menor riesgo de desenganche educativo. Al tiempo que hemos identificado dos vías de acceso diferenciado a la FPB en la que se combinan, o no, los malos resultados académicos con conductas disruptivas en el aula, siendo las chicas especialmente minoritarias en éste último canal de acceso.

Por otro lado, hemos podido constatar que el profesorado entrevistado, por una parte, asume el discurso políticamente correcto de la igualdad de género, es decir, afirman en todo momento la ausencia de diferencias de capacidades entre chicas y chicos para realizar determinadas tareas. Por otra parte, asume de manera irrenunciable el discurso de los gustos individuales, con lo que, ante itinerarios feminizados y masculinizados, justifican esta segregación en base a los gustos y preferencias personales, razones de tipo expresivo, del estudiantado. Asumir que se trata de preferencias y gustos personales diluye el contenido estructural de la segregación de los estudios de FPB con lo que también se aminora la importancia de incidir en ésta de manera estructural y por ello, las medidas y acciones que desde los centros se llevan a cabo se centran en promocionar actitudes y posicionamientos individuales. Además, encontramos un elemento fundamental que viene a dificultar la implementación de medidas firmes por la igualdad y la diversidad de género: la priorización del reenganche educativo entre un colectivo de estudiantes que han mostrado unas trayectorias en las que la identificación con los estudios reglados es muy débil o incluso inexistente. Todo ello mediatizado por los escasos recursos personales y económicos con los que cuentan los centros al tiempo que la consideración por parte de los agentes sociales implicados de su limitada incidencia en el contexto social en el que se insieren.

En definitiva, el análisis desde una perspectiva de género de las narraciones de los docentes que desarrollan tareas de dirección y orientación en centros en los que se imparte Formación Profesional Básica nos ha permitido observar el elevado grado de interiorización del género, desde un marco discursivo normativo y binario, en la construcción de su mirada sobre las trayectorias y decisiones formativas del estudiantado. Una interiorización que influye directamente en la escasa implementación de medidas para transformar esta realidad más allá de la superficie y en consecuencia en la perdurabilidad de las marcadas asimetrías de género que existen en la FPB y con ello también en su futuro laboral. Por todo ello no solo cabe investigar la elección y orientación laboral que reciben estas jóvenes, sino avanzar en orientarlas a sectores con mayores posibilidades futuras de movilidad social como el tecnológico. Ello no solo es responsabilidad de las mujeres y sus elecciones, sino, sobre todo, de las opciones que les brindamos. En este sentido, cabe investigar para reformular títulos, replantear el marketing educativo, generar nuevos discur- 
sos, repensar los currículums y los contenidos, repensar la orientación educativa y profesional, revisar los requisitos y formatos pedagógicos y académicos para que dejen de ser ciegos al género y se orienten a mayores derechos, autonomía y oportunidades para las mujeres.

Con todo, al acabar este análisis se abre un importante elemento de reflexión que será necesario profundizar en investigaciones futuras. El mantenimiento de la institución educativa como institución de género (Connell, 2001) conlleva claramente la reproducción de la desigualdad de género en nuestra sociedad. Su posición minoritaria en el ciclo de FPB interviene en su invisibilidad pero también en la penalización de aquellas que sí que están. Una penalización doble: a través de la consideración negativa de su conducta y opciones, más que en el caso de los chicos, y a través de una más que probable precarización de sus opciones laborales (y por tanto vitales) futuras. Recordemos se trata de un ciclo formativo que proviene de una diversificación curricular en la etapa obligatoria con el fin de evitar el abandono educativo temprano, por tanto con escasa consideración tanto académica como laboral, así como social. Y además estas chicas se concentran en unas pocas familias profesionales precisamente porque son las que no suponen ninguna ruptura con las expectativas de género que se les atribuye: cuidado de la imagen, pero también tareas administrativas o comerciales o de confección. Formación que, en general, ofrece escasas oportunidades para acceder a puestos de trabajo con suficientes condiciones de calidad y estabilidad. Por tanto, estaríamos ante una doble precarización de las chicas: por estudiar una FPB y por hacerlo en familias profesionales con escasas oportunidades laborales. La desigualdad de género se mantiene y agrava a través de itinerarios formativos que desembocan en posiciones labores claramente marcadas por género.

\section{Referencias bibliográficas}

Abiétar-López, Míriam; Navas-Saurin, Almudena; Marhuenda-Fluixá, Fernando (2015): “Aportaciones desde la justicia social para una educación justa: La identidad pedagógica en formación profesional básica”. Revista Internacional de Educación para la Justicia Social (RIEJS), 4 (2), 145-161.

Acker, Joan (1992): "From sex roles to gendered institutions". Contemporary Sociology, 21 (5), 565-569. DOI: https://doi.org/10.2307/2075528.

Barberà, Esther; Candela, Carlos; Ramos, Amparo (2008): "Elección de carrera, desarrollo profesional y estereotipos de género”. Revista de Psicología Social, 23 (2), 275-285. DOI: https://doi. org/10.1174/021347408784135805.

Bernad, Joan Carles; Molpeceres, María Ángeles (2006): “Discursos emergentes sobre la educación en los márgenes del sistema educativo". Revista de educación, 341, 149-170.

Bonal, Xavier; Tarabini-Castellani, Aina; Verger, Antoni (2015). "La nova política educativa i les desigualtats". Nous Horitzons, 209, 16-21.

Bourdieu, Pierre (2000): "Sobre el poder simbólico" en Pierre Bourdieu: Intelectuales, política y poder. Buenos Aires: UBA/Eudeba.

Bourdieu, Pierre; Passeron Jean Claude (2001). La reproducción: elementos para una teoría del sistema de enseñanza. Madrid: Editorial Popular. 
Burchell, Brendan; Hardy, Vincent; Rubery, Jill; Smith, Mark (2015). A New Method to Understand Occupational Gender Segregation in European Labour Markets. Brussels: European Commission. DOI: https://doi. org/10.2838/748887.

Cáceres, Juan I.; Escot, Lorenzo; Sainz, Javier (2004). La segregación ocupacional sectorial de la mujer en el mercado de trabajo español. Documento de Trabajo. Facultad CC.EE: Universidad Complutense de Madrid.

Cacheiro González, Maria L.; García García, Francisco; Moreno Guerrero, Antonio J. (2016): "Las TIC en los programas de Formación Profesional Básica en Ceuta”. Apertura, 7 (2), 132-151.

Cantalapiedra García, Vivianne; Marginet Flinch, Mónica; Almeda Samaranch, Elisabet (Coords.) (2019) Desigualdades y Cárceles de mujeres. Voces y debates desde el feminismo. Colección: Mujeres y Sistema Penal. Número 04. Barcelona. Copalqui Editorial.

Casado Martínez, Carlos; Meneses Naranjo, Julio; Sancho Vinuesa, Teresa (2016). “¿Cómo ven los alumnos de primaria la profesión informática? Influencia del género y la percepción de su capacidad”. PíxelBit. Revista de Medios y Educación, 49, 149-161. DOI: https://doi.org/10.12795/pixelbit.

Castaño, Cecilia (2005). Las mujeres y las tecnologías de la información: Internet y la trama de nuestra vida. Madrid: Alianza.

Castaño, Cecilia; Webster, Juliet (2014). Género, ciencia y tecnologías de la información. Madrid: Editorial Aresta.

Cebrián, Inmaculada; Moreno, Gloria (2018). "Desigualdad de género en el mercado laboral”. Panorama Social, 27, 47-63.

Connell, Raewyn (2001). "Educando a los muchachos: Educando a los muchachos: la educación física mixta en clave de género”. Nómadas, 14, 156-173.

Consejo Económico y Social-CES (2016): “La participación laboral de las mujeres en España”. Informe 05/2016. http://www.ces.es.

Durán, Maria Ángeles (2018). La riqueza invisible del cuidado. València: Publicacions de la Universitat de València.

EIGE (2017). Gender, Skills and Precarious Work in the EU. Vilnius: (European Institute for Gender Equality) EIGE. DOI: https://doi.org/10.2839/62350.

Evans, Karen (2006): “Achieving equity through 'gender autonomy': the challenges for VET policy and practice". Journal of Vocational Education and Training, 58 (4), 393-408. DOI: https://doi. org/10.1080/13636820601005453

Fernández Casado, Ana B.; Ibáñez Pascual, Marta (2018): "Más mujeres en los estudios de Informática: una propuesta desde el departamento de formación y orientación laboral". Revista de la Asociación de Sociología de la Educación (RASE), 11 (1), 116-134. DOI: https://doi.org/10.7203/RASE.11.1.10624.

Frutos, Lola (2010). "Identidad de género en las trayectorias académicas y profesionales de las mujeres". Revista de la asociación de sociología de la Educación, 3 (3), 336-356. 
González, Ana M.; Vergés, Núria, Martínez, J. S. (2017): "Las mujeres en el mercado de trabajo de las tecnologías”. REIS: Revista española de investigaciones sociológicas, 159, 73-90. DOI: http:/ /dx.doi.org/10.5477/ cis/reis.159.73.

Høst, Hakon; Seland, Iduun; Skålholt, Asgeir. (2015). "Gender Policies Meet VET Practices - The Case of Health and sSocial Care in Norway". Journal of Vocational Education \& Training, 67 (1), 109-126. DOI: http://dx.doi.org/10.1080/13636820.2014.958869.

Ibáñez, Marta (2008): "La segregación ocupacional por sexo a examen. Características personales, de los puestos y de las empresas asociadas a las ocupaciones masculinas y femeninas”. REIS: Revista Española de Investigaciones Sociologicas, 123, 87-122.

INE (2018). Mujeres y hombres en España.

Jackson, Carolyn (2006): “Wild' Girls? An Exploration of 'Ladette' Cultures in Secondary Schools". Gender and Education, 18 (4), 339-360. DOI: http:/ /dx.doi.org/10.1080/09540250600804966.

MECD (2018). Las cifras de la educación en España (en línea). http:/ / www.mecd.gob.es, consultado en enero 2019.

Millenaar, Verónica (2014): “Trayectorias de inserción laboral de mujeres jóvenes pobres: el lugar de los programas de Formación Profesional y sus abordajes de género”. Trabajo y sociedad, 22, 325-339.

Niemeyer, Beatrix; Colley, Helen (2015): "Why Do We Need (Another) Special Issue on Gender and VET? Editorial”. Journal of Vocational Education and Training, 17 (1), 1-10. DOI: https://doi.org/10.10 80/13636820.2014.971498.

Martínez, José Saturnino; Merino, Rafael (2011): "Formación Profesional y desigualdad de oportunidades educativas por clase social y género". Témpora, 14, 13-37.

Merino, Rafael (2012): “La formación profesional en los itinerarios formativos y laborales de los jóvenes: las leyes proponen y los jóvenes disponen”. Revista de Sociología de la Educación (RASE), 5 (3), 503-512.

Merino, Rafael; Martínez, José Saturnino (2012): "La formación profesional y la desigualdad social". Cuadernos de Pedagogía, 425, 34-37.

Obiol-Francés, Sandra (2017): "Anar a la universitat, una decisió només individual? El paper de la familia" en Alícia Villar-Aguilés (coord.): La decisió d'anar a la universitat. Processos d'orientació i transicions educatives en temps d'incertesa. València: Editorial Tirant lo Blanch.

Obiol-Francés, Sandra; Villar-Aguilés, Alícia (2016): "Másteres masculinizados, másteres feminizados. La persistencia de la segregación de género en la formación de postgrado en España”. Comunicación XII Congreso Español de Sociología.

OIT (2018). Global Wage Report 2018/19: What lies behind gender pay gaps (en línea). https://www.ilo. org/global/publications/books/WCMS_650553/lang--en/index.htm.

OECD (2018). Education at a Glance 2018. Paris: OECD Indicators. DOI: https://doi.org/10.1787/ eag-2018-en, consultado en enero 2019. 
Roca Cobo, Enrique (2010): "El abandono temprano de la educación y la formación en España”. Revista de Educación, núm. extraordinario.

Sainz, Milagros (2011): “Factors Which Influence Girls' Orientations to ICT Subjects in Schools. Evidence from Spain”. International Journal of Gender, Science and Technology, 3 (2), 387-406.

Sainz, Milagros; Pálmen, Rachel; García-Cuesta, Sara (2012). "Parental and Secondary School Teachers' Perceptions of ICT Professionals, Gender Differences and Their Role in the Choice of Studies". Sex roles, 66 (3-4), 235-249. DOI: https://doi.org/10.1007/s11199-011-0055-9.

Subirats, Marina (2016): "De los dispositivos selectivos en la educación: el caso del sexismo". Revista de la Asociación de Sociología de la Educación (RASE), 9 (1), 22-36.

Subirats, Marina; Tomé, Amparo (2007). Balones fuera: reconstruir los espacios desde la coeducación. Barcelona: Octaedro.

Torns, Teresa; Recio, Carolina (2012): "Las desigualdades de género en el mercado de trabajo: entre la continuidad y la transformación”. Revista de economía crítica, 14 (segundo semestre), 178-202.

Trotter, Latonya J. (2017). "Making A Career: Reproducing Gender within a Predominately Female Profession”. Gender \& Society, 31 (4), 503-525. DOI: https://doi.org/10.1177/0891243217716115.

Vázquez-Cupeiro, Susana (2015): “Ciencia, estereotipos y género: una revisión de los marcos explicativos”. Convergencia, 22 (68), 177-202.

Vergés Bosch, Núria (2012). "De la exclusión a la autoinclusión de las mujeres en las TIC. Motivaciones, posibilitadores y mecanismos de autoinclusión". Athenea Digital. Revista de pensamiento e investigación social, 12 (3), 129-150. DOI: https://doi.org/10.5565/rev/athenead/v12n3.1042.

Villar-Aguilés, Alícia (coord.) (2017). La decisió d'anar a la universitat. Processos d'orientació i transicions educatives en temps d'incertesa. València: Tirant lo Blanch.

Villar-Aguilés, Alícia; Hernàndez, Francesc J. (2014). "University Transitions and Gender: From Choice of Studies to Academic Career Development". Policy Futures in Education, 12 (5). DOI: https://doi. org/10.2304/pfie.2014.12.5.633.

Villar-Aguilés, Alícia; Obiol-Francés, Sandra (2020) “Itinerarios generizados en Formación Profesional Básica. Resultados de un estudio en centros educativos valencianos”. Revista Recerca. Artículo entregado para publicación. 


\section{Annexo}

Tabla I. Centros analizados en el análisis cualitativo

\begin{tabular}{|c|c|c|c|c|c|c|}
\hline CÓDIGO & $\begin{array}{c}\text { TIPO } \\
\text { CENTRO }\end{array}$ & TITULARIDAD & $\begin{array}{l}\text { TAMAÑO } \\
\text { HÁBITAT }\end{array}$ & $\begin{array}{l}\text { ITINERARIO } \\
\text { FPB }\end{array}$ & $\begin{array}{c}\text { OTROS } \\
\text { ITINERARIOS }\end{array}$ & $\begin{array}{l}\text { TRABAJO } \\
\text { DE CAMPO }\end{array}$ \\
\hline $\mathrm{Cl}$ & $\begin{array}{l}\text { Instituto Educación } \\
\text { Secundaria }\end{array}$ & Público & 5.000-10.000 hab. & $\begin{array}{c}\text { Imagen personal-Peluquería y estética } \\
\text { Agrara-Agro jardinería y composiciones florales }\end{array}$ & $\begin{array}{c}\text { ESO } \\
\text { Bachillerato } \\
\text { Ciclos formativos }\end{array}$ & $\begin{array}{c}\text { Dirección-Orientación } \\
4 \text { hombres } \\
3 \text { mujeres } \\
2 \text { GD 2 ESO }\end{array}$ \\
\hline $\mathrm{C2}$ & $\begin{array}{l}\text { Centro integrado de } \\
\text { Formación Profesional }\end{array}$ & Público & $50.000-80.000$ hab. & $\begin{array}{l}\text { Hostelería y turismo-Cocina y restauración } \\
\text { Imagen personal-Peluquería y estética }\end{array}$ & $\begin{array}{l}\text { Ciclos formativos } \\
\text { Ciclos } 2^{0} \text { oportunidad }\end{array}$ & $\begin{array}{l}\text { Dirección-Orientación } \\
5 \text { mujeres } \\
1 \text { hombre }\end{array}$ \\
\hline C3 & $\begin{array}{l}\text { Instituto Educación } \\
\text { Secundaria }\end{array}$ & Público & $80.000-100.000$ hab. & $\begin{array}{c}\text { Electricidad y electrónica-Electricidad y electrónica } \\
\text { Transporte y mantenimiento } \\
\text { de vehículos-Mantenimiento de vehículos }\end{array}$ & $\begin{array}{c}\text { ESO } \\
\text { Bachillerato } \\
\text { Ciclos formativos }\end{array}$ & $\begin{array}{l}\text { Dirección-Orientación } \\
1 \text { mujer } \\
11 \text { hombres }\end{array}$ \\
\hline C4 & $\begin{array}{l}\text { Centro Privado Educación } \\
\text { Infantil, Primaria y } \\
\text { Secundaria }\end{array}$ & Concertado & 50.000-80.000 hab. & $\begin{array}{l}\text { Madera, mueble y corcho-Carpintería y mueble } \\
\text { Imagen personal- Peluquería y estética }\end{array}$ & $\begin{array}{l}\text { Educación Infantil } \\
\text { Educación Primaria } \\
\text { ESO }\end{array}$ & $\begin{array}{l}\text { Dirección-Orientación } \\
3 \text { hombres } \\
5 \text { mujeres }\end{array}$ \\
\hline$C 5$ & $\begin{array}{l}\text { Instituto Educación } \\
\text { Secundaria }\end{array}$ & Público & 10.000-25.000 hab. & $\begin{array}{l}\text { Imagen personal- Peluquería y estética } \\
\text { Electricidad y electrónica-Electricidad y electrónica }\end{array}$ & $\begin{array}{c}\text { ESO } \\
\text { Bachillerato } \\
\text { Ciclos formativos } \\
\text { Bachillerato } \\
\text { Ciclos formativos }\end{array}$ & Dirección-Orientación \\
\hline
\end{tabular}

\section{Notas biográficas}

Sandra Obiol Francés es profesora contratada doctora del Departamento de Sociología y Antropología Social de la Universitat de València. Doctora por la Universitat Autònoma de Barcelona, miembro del grupo de investigación IPRODES (Universitat de València) y COPOLIS (Universitat de Barcelona) además del grupo de innovación docente CEFOCID-COPOLIS. Sus principales líneas de investigación son el análisis del cambio familiar desde una perspectiva de género, los procesos de precarización laboral y, más recientemente, la relación entre trabajo de cuidados y trabajo remunerado, en especial, en profesiones cualificadas. En relación a estos intereses ha participado en numerosos proyectos de investigación de los que se han derivado diversas publicaciones en revistas académicas, pero también ha participado activamente en actividades y publicaciones de divulgación científica. Ha realizado estancias de investigación en Estados Unidos, Inglaterra, Alemania y Argentina.

Elisabet Almeda Samaranch es Catedrática de Sociología de la Universitat de Barcelona, integrante del Grupo COPOLIS y de la Fundación Adalquí (Argentina). Docente, investigadora y activista feminista, ha hecho investigación y docencia en tres grandes ámbitos: sistemas de ejecución penal femenina (control social, delincuencia femenina, cárceles de mujeres), cambios y políticas familiares (familias monoparentales, transformaciones y políticas familiares comparadas) y memoria e identidad durante la dictadura franquista (cotidianidades y resistencias). Ha publicado los resultados de sus investigaciones tanto en publicaciones de carácter académico como de divulgación social y en todos estos campos ha impartido docencia en universidades de Catalunya, España, América del sur, América central, Norteamérica y Eu- 
ropa. También ha fundado y participado de varias redes, comisiones y colectivos, entre las que destacan la Red Temática Internacional de Investigación sobre Familias, el Colectivo de mujeres presas Cassandra y la Red Temática Internacional sobre Género y Sistema penal.

Dino Di Nella es es docente ordinario adjunto en la Universidad Nacional de Río Negro, abogado por la Universidad Nacional de la Plata (Argentina), operador comunitario, magister en «Políticas de igualdad de género: agentes de igualdad» por la Universidad Autónoma de Barcelona, y doctor en Sociología por la Universidad de Barcelona. Integra el Centro de Investigación en Derecho Crítico (CIDERCRIT) de la Universidad Nacional de La Plata, el Grupo de Estudios Copolis-Adalquí de la Sede Atlántica de la Universidad Nacional de Río Negro, y la línea de actuación sobre «Control Social y Derechos Humanos», del Grupo Interuniversitario de Investigación, Formación y Extensión Universitaria «COPOLIS. Bienestar, Comunidad y Control Social», de la Universidad de Barcelona.

ORCID: https://orcid.org/0000-0003-1606-7712.

Nuria Pumar Beltrán es profesora titular de Derecho del Trabajo del Departamento de Derecho Privado de la Universidad de Barcelona (UB). Doctora en Derecho por la Universidad Pompeu Fabra (1999) con mención de derecho europeo. En la actualidad coordina el Máster d'Estudis de Dones, Gènere i Ciutadania y ha dirigido el Instituto Interuniversitario impulsor de este máster. Es miembro del grupo de investigación COPOLIS y sus líneas principales de investigación versan sobre el tema de igualdad y la acción positiva en materia de Derecho del trabajo y protección social, así como sobre las políticas de empleo en la Unión Europea y en España.

Elisabet Almeda Samaranch es Abogada y Profesora asociada en la Universidad Pompeu Fabra de Derecho del Trabajo y la Seguridad Social. Investigadora principal del proyecto EEGrants «Un futuro sin brecha». Entre el año 2010 a 2018 Directora de la Fundación María Aurelia Capmany https://www. fmac.org/qui-som/.

Núria Vergés Bosch es profesora lectora del Departamento de Sociología de la Universidad de Barcelona. Forma parte del Grupo consolidado de investigación COPOLIS, del de innovación docente CEFOCID-COPOLIS, así como del Instituto Interuniversitario de Género de Catalunya (IIEDG). Doctora en Sociedad de la Información y del Conocimiento (In3-UOC), Máster en Políticas Públicas y Sociales (UPF), licenciada en Ciencias Políticas (UAB). Sus investigaciones tienen que ver con 5 líneas de investigación principales: la relación género y sociedad de la información; las políticas públicas, familiares y el Estado del Bienestar; la memoria colectiva; las violencias machistas y las metodologías de investigación feminista. Ha participado en más de 50 investigaciones y tiene publicaciones de impacto de todas ellas. Es miembra de varias entidades y colectivas de género y de experimentación TIC. Destaca la colectiva Donestech, de mujeres y nuevas tecnologías, la asociación Alia de mujeres para la investigación y la acción y SIMREF de metodologías feministas.. 
Alícia Villar Aguilés es profesora de sociología del Departamento de Sociología y Antropología Social y adscrita al Instituto de Creatividad e Innovaciones Educativas de la Universitat de València. Sus líneas de investigación se centran, principalmente, en el análisis sociológico de la educación y en el análisis sociológico de la conciliación del tiempo personal, familiar y laboral. Ha publicado trabajos sobre decisiones y trayectorias educativas, elecciones y abandono de estudios y universidad. También le interesa el estudio de la desigualdad desde una perspectiva feminista interseccional. Forma parte del Comité de Sociología de la Educación de la Federación Española de Sociología, de la Associació Valenciana de Sociologia y del grupo de trabajo CLACSO-Universidades y Políticas de Educación Superior. Participa en el Lab-Feminisme de la Fundació Nexe. Ha sido Erasmus Teacher en la Universidad de Coímbra (Portugal) y ha realizado estancias de investigación en Portugal, Alemania y Brasil. 